\title{
NOTAS SOBRE LUCHAS DE CLASE Y LUCHAS SOCIO-POLÍTICAS EN NUESTRA AMERICA EN EL CONTEXTO ACTUAL ${ }^{1}$
}

Notes on class struggles and socio-political struggles in Our America in the current context

CASAS, Alejandro Pablo ${ }^{2}$

Resumen: Se busca problematizar teórica e históricamente las categorías de luchas de clase y luchas socio-políticas, atendiendo a sus múltiples conexiones y especificidades, con particular interés para Nuestra América y los sujetos colectivos populares en el contexto del capitalismo actual. Se intenta abordar un terreno teórico que ha sido imbuído por las proposiciones de perspectivas positivistas, evolucionistas y/o posmodernas, y que ha atravesado el debate sobre los movimientos sociales. Al otorgarse prioridad exclusiva a la clase por sobre otras luchas sociales, o al colocar a las mismas en un pie de igualdad o de indeterminación, o al forzar un enfoque cultural/identitario restringido, no se da cuenta de la complejidad de la cuestión. Para ello se recurre a un repaso teórico, con particular interés en algunas perspectivas del materialismo histórico, al mismo tiempo que se abordan algunas configuraciones modernas de las luchas sociales y de clase en América Latina y el Caribe.

Palabras claves: Materialismo histórico. Luchas de clase. Luchas socio-políticas. Sujetos colectivos populares. Nuestra América.

Abstract: The purpose is to problematise theoretical and historically, the categories of class struggles and socio-political struggles, attending on its multiple connections and specificities, with a particular interest in Our America and the popular collective subjects in the context of the current capitalism. Attempts to address a theoretical ground that has been imbued by the propositions of positivist, evolutionary and/or post-modern perspectives, and that has gone through the debate on social movements. To the priority given exclusively to the class by other social struggles, or by placing them in terms of equality or indeterminacy, or forcing a cultural/identity approach restricted, does not realize the complexity of the issue. For this purpose, a theoretical review, with particular interest in some perspectives of historical materialism, are used at the same time addressed some modern configurations of social struggles and class in Latin America and the Caribbean.

Keywords: Historical materialism. Class struggles. Socio-political struggles. Popular collective subjects. Our America.

\footnotetext{
${ }^{1}$ Recebido em: 16 mai. 2018. Aprovado em: 10 ago. 2018.

${ }^{2}$ Asistente Social Universitario (Universidad de la República), Magister y Doctor en Servicio Social por la Universidad Federal de Rio de Janeiro, Brasil. Docente investigador a tiempo completo del Departamento de Trabajo Social de la Facultad de Ciencias Sociales de la Universidad de la República, Uruguay. Investigador en temáticas de movimientos sociales, pensamiento crítico en América Latina y Trabajo Social. Correo: alejandro.casas@cienciassociales.edu.uy
} 


\section{Introducción}

Se trata de apuntar a una primera aproximación de la problematización teórica de las categorías de luchas de clase y luchas socio-políticas, así como identificar diversas conexiones, articulaciones y especificidades, atendiendo a algunas particularidades de los sujetos colectivos y las luchas populares en Nuestra América en el contexto actual del capitalismo tardío.

Una preocupación central dice con intentar clarificar conceptualmente un terreno teórico que ha sido imbuído por las proposiciones de ciertas perspectivas economicistas, neopositivistas y/o posmodernas; tanto sea que la clase (y las luchas asociadas) se diluya en una concepción que privilegia el orden social y su reproducción (cuando no se la niega directamente), o se de prioridad exclusiva a la clase por sobre otras luchas o demandas sociales, o porque se coloquen a las mismas en un pie de igualdad o de indeterminación (entendidas como la juxtaposición o agregación de demandas de clase, género, étnico/raciales, anticoloniales o nacional-populares, etc.), no parece darse cuenta de la complejidad de la cuestión.

Al mismo tiempo parte de la literatura que ha abordado la cuestión de los movimientos sociales ha tendido a facilitar análisis reduccionistas, cuando por ejemplo se contraponen las nuevas demandas que emergen en dichas luchas contra las "tradicionales" y "vetustas" luchas de los sindicatos o partidos obreros, cuando se recurre a abordajes que parten de una lectura desde el "rational choice" y sus abordajes generalmente centrados en el individualismo metodológico ${ }^{3}$, o cuando se coloca el foco en demandas por reconocimiento o de tipo cultural dejando de lado su vinculación con otros procesos económico/políticos, etc. Para ello apelamos también al uso de la categoría de sujetos colectivos populares.

Para ello se recurre a una primera revisión de la literatura en el campo del marxismo original y del pensamiento crítico en general en torno a estos temas, para poder dar cuenta de las necesarias mediaciones para la comprensión/explicación de estos procesos sociales en Nuestra América, atendiendo también a las particularidades de tiempo y espacio que las mismas suponen, tomando en cuenta los distintos niveles de llamado "marxismo analítico". (cf. Bensaïd, 2003, p 187- 297) 
abstracción que dichas categorías implican.

Se parte de una recuperación de algunas claves interpretativas del pensamiento de Marx y de algunas tradiciones del materialismo histórico (o de la "filosofía de la praxis") así como de algunos aportes del pensamiento crítico y el marxismo en América Latina, atendiendo a sus aportes para pensar la cuestión en un plano más conceptual y socio/histórico. Por otra parte se recupera la historicidad de diversas luchas de clases y socio-políticas en Nuestra América, atendiendo a sus configuraciones modernas, y ensayando algunas interpretaciones al respecto.

Partimos de la hipótesis de que es necesario problematizar esta falsa dicotomía (o aislamiento) que a veces se presenta entre luchas de clase y luchas socio-políticas ${ }^{4}$, al mismo tiempo que para incorporar también la cuestión de la lucha ético-cultural, como cuestiones que entendemos deben pensarse de forma entrelazada, aún sin anular sus especificidades. Al mismo tiempo parece necesario distinguir un nivel más abstractoesencial, y otro más histórico-concreto, donde van aparecer un conjunto de mediaciones y de diversas luchas sociales que no pueden desconectarse del plano de la lucha de clases, al menos en el plano del análisis y la reflexión teórica.

\section{Clase, lucha de clases y luchas socio-políticas en el materialismo histórico: una primera aproximación}

En primer lugar parece necesario discriminar y vincular la categoría de "clase social" de la de "lucha de clases". La clase ha sido una categoría ampliamente debatida al interior de las ciencias sociales. Como es sabido, hablar teóricamente de clases sociales no implica posicionarse necesariamente desde una perspectiva inspirada en el materialismo histórico. La existencia de clases sociales, en el marxismo "original” de Marx, está articulada, de forma sistemática, a otro conjunto de categorías que se vinculan a su configuración moderna en el marco de la interacción dialéctica entre ser social y mundo

\footnotetext{
${ }^{4}$ En este sentido es interesante recuperar la crítica de Mészáros, en tanto que existe una crítica muy fuerte a la separación entre la lucha política, y aquella vinculada a la reproducción de la vida material, que caracteriza en buena medida una división del trabajo entre partidos de izquierda (comunistas, socialistas y otros), y los sindicatos de trabajadores. La reconstitución de dicha unidad es la característica esencial definidora del modo socialista de control sociometabólico (Mészáros, 2002, p. 30-1).
} 
natural en el orden burgués, desde una perspectiva de totalidad. Así la clase se vincula a otras como trabajo, explotación, plusvalor, mercancía, dinero, capital y acumulación de capital, Estado, revolución, hegemonía (incorporada por Lenin y Gramsci), entre otras.

Son varias las indicaciones teóricas (en sus trabajos más propiamente teóricos como El capital, entre otros varios) y socio/históricas (en sus trabajos más histórico/políticos más "concretos" como El 18 Brumario de Luis Bonaparte, Las luchas de clases en Francia de 1848 a 1850 o La guerra civil en Francia, o incluso en el propio capítulo XXIV del libro I de El capital sobre "La llamada acumulación originaria" entre otros) que Marx desarrolla en torno a la categoría de clase social, más allá de que el manuscrito del Libro III del Capital se interrumpe justamente cuando comienza a hablar de las clases.

Por otra parte más que propiamente la clase en sí, la categoría más abarcativa parece ser la de lucha de clase; es recordada una de las frases iniciales del Manifiesto Comunista cuando sostiene junto con Engels que "la historia de todas las sociedades hasta nuestros días es la historia de las luchas de clases" (1969, p. 35). La lucha de clases en Marx implica la existencia de otras clases con intereses y proyectos antagónicos, además de la existencia de intereses comunes a su interior, asi como de otros aspectos culturales y de vida en común que unifican (o pueden hacerlo) a las mismas. Dice Marx en la Miseria de la Filosofía que "la lucha de clase contra clase es una lucha política". Marx ilustra por ejemplo que la lucha por la reducción de la jornada de trabajo en una empresa es una lucha económica; mientras que la lucha por que se decrete la ley de la jornada de trabajo de 8 horas es un movimiento político (en Carta a Bolte) (cf. Badaró, 2012, p. 62-3). Engels hablará por su parte de tres niveles de lucha articulados: económica, ideológica y política (teniendo en cuenta como horizonte la lucha por la apropiación y transformación del aparato estatal). ${ }^{5}$

Daniel Bensaïd nos brinda una lectura compleja de la formación de las clases en el sistema capitalista, a partir de un análisis de la complementariedad existente en Marx

\footnotetext{
${ }^{5}$ Si se quiere retomar una conceptualización de las clases sociales, aún tomando en cuenta las reservas del propio Marx en relación a un método de investigación que reniega de las definiciones "cerradas" (como desarrollaremos más adelante), se puede ir al propio Lenin: "Las clases son grandes grupos de hombres que se diferencian entre sí por el lugar que ocupan en un sistema de producción social históricamente determinado, por las relaciones en que se encuentran con respecto a los medios de producción (relaciones que las leyes refrendan y formulan en su mayor parte), por el papel que desempeñan en la organización social del trabajo, y, consiguientemente, por el modo y la proporción en que perciben la parte de riqueza social de que disponen" (Lenin apud Bensaïd, 2003, p. 268)
} 
entre los tres libros de El Capital. Si el libro I se centra en la producción (en un nivel más abstracto), el segundo lo hace en el proceso de circulación, y el tercero en el nivel de la reproducción en su conjunto. Hay allí un movimiento de lo "abstracto a lo concreto" - a tono con los desarrollos realizados por Marx (1984), en Los Grundrisse de 1857-8, por ejemplo en la sección sobre el método de la economía política (cf. Dussel, 1985)-, y un proceso que sólo que se completa en términos de la lógica general de construcción de dicha crítica y marco categorial ${ }^{6}$. Dice Bensaïd: "La oposición simple entre trabajo asalariado y capital no se sitúa, en Marx, en el nivel de la formación social. Reside en el primer nivel de la abstracción determinada, el de la esfera de la producción" (2003, p. 156); "la contradicción, estática en el nivel de la producción, se resuelve en la circulación y la reproducción". En el libro primero se trata de la reproducción simple, el libro segundo plantea que "la reproducción simple es abstracción", y en ese sentido, que en el sistema capitalista "la falta de acumulación o de reproducción a escala ampliada es una hipótesis extraña"; en el tercer libro se referirá al proceso de producción global (2003, p. 369), por lo tanto en ese sentido su análisis en los tres libros se irá "concretizando". El nivel de la "formación social" (en términos de Lenin) ya es más concreto, y más aún lo será el nivel del análisis de la coyuntura o de "situaciones concretas", y por lo tanto según el método de Marx, puede ser entendido como la "síntesis de múltiples determinaciones" (Marx, 1984). Pero lo más concreto no es lo empírico, sino una construcción que se mueve en el plano del pensamiento y que reproduce el movimiento de la totalidad concreta. ${ }^{7}$

\footnotetext{
${ }^{6}$ Recurriendo explícitamente el método hegeliano desarrollado por Marx dice Bensaïd: "Marx reivindica explícitamente esta lógica dinámica de la determinación, opuesta a la lógica estática y clasificatoria de la definición. [...] La relación de lo abstsracto con lo concreto procede directamente de la determinación. Al contrario de la abstracción unilateral (especulativa) la abstracción determinada permite que lo que una feliz metáfora cinematográfica designa como "puntualización histórica de las categorías", a través de su "conexión orgánica interna". Afirmativa, la impaciente definición satisface una sed de positividad inmediata. En el paciente trabajo de lo negativo, la determinación llega por el "ausentismo" o la supresión de la carencia. [...] La "circularidad del método de El capital es un ejemplo de "la circularidad propia de todo conocimiento racional, de toda teoría" [citando a Marx]. La circularidad del conocimiento reproduce la circularidad de su objeto. De metamorfosis en permutaciones, la mercancía abandona un ropaje para saltar inmediatamente al siguiente, de modo que "cada momento aparece como punto de partida". El círculo abierto de la reproducción ampliada permite la superación de la triste repetición dentro de lo aleatorio circunstancial." (Bensaïd, 2003, p. 366-9).

${ }^{7}$ Como indica Bensaïd, en el libro primero la "relación de explotación", si bien esencial, "entre trabajo asalariado y capital no es más que la primera y más abstracta de sus determinaciones". (2003, p. 166). El libro segundo trata de las relaciones de clase en la unidad de la producción y la circulación, desarrollando "el ciclo de las metamorfosis del capital" (2003, p. 168): "Propia de la esfera de la circulación, la relación de compra y venta de la fuerza de trabajo no es menos constitutiva de la relación de clase que la relación de explotación en el libro primero" (2003, p. 169) En este caso se agrega una nueva determinación, la del "trabajo productivo" o indirectamente productivo (2003, p. 170). En el libro tercero, en el marco del análisis de la producción y reproducción en su conjunto, recién se plantearán otros determinaciones para el análisis
} 
No podemos profundizar aquí en esta argumentación sobre las distintos niveles de determinaciones de las clases en El capital, y en general en el conjunto de la obra de Marx, lo que excedería con creces la intención y posibilidades de este artículo. Sin embargo queríamos dejar planteada la complejidad que esta cuestión tuvo en el fundador del materialismo histórico, y que por lo general, no es recuperada en algunos análisis más mecanicistas o deterministas de su obra, tanto identificándose dentro de sus filas como fuera o contra ellas. Muchas veces se confunden niveles más "esenciales" con niveles más "concretos" de análisis en la obra del propio Marx, y ello lleva a errores teóricos y metodológicos evidentes, teniendo repercusiones en las cuestiones que intentamos analizar aquí. Por otro lado no olvidemos que esto también ha estado presente en el análisis del capitalismo en América Latina, dando lugar a muchos malentendidos.

Pero además, como indica el propio Bensaïd, “En el campo específico de lo político, las relaciones de las clases adquieren un grado de complejidad irreductible al antagonismo bipolar que sin embargo las determina" (2003, p. 176, destacado personal). Aparecen el Estado, fracciones de clase y grupos que no tienen una representación directa de sus intereses en el plano político o del Estado, sectores medios, alianzas de clases, ideologías y formas culturales, relaciones de dependencia entre naciones a escala internacional, el mercado mundial y la división internacional del trabajo, etc; aunque sin el tratamiento más sistemático que el propio Marx se había planteado desarrollar en varios de sus planes y no llegó a concretar en vida. (cf. Rosdolsky, 1978)

También debemos destacar, enfatizando sobre todo el llamado "viraje" históricopolítico de Marx en los últimos años de su vida, vinculado sobre todo a la "cuestión rusa", el interesante abordaje que realiza Enrique Dussel (1990), donde se cuestionan algunos rasgos supuestamente eurocentristas en Marx. Con una excelente documentación, el autor analiza el diálogo y la sensibilidad de Marx para con los populistas rusos (narodniki). Demuestra las distintas respuestas que dieron Marx y Engels en términos de la posibilidad de la revolución en Rusia (divergiendo en si debía estar subordinada o no al triunfo de la revolución en Europa occidental), que tendrán por su lado repercusiones importantes en la conducción y en algunas concepciones que se volverán dominantes en el seno de la II, y

de las clases. Las determinaciones parciales de las clases en los libros anteriores, se integran desde ese momento al "movimiento de conjunto de la competencia, de la persecusión de la tasa de ganancia, de la especialización funcional de los capitales, y de la distribución del ingreso." (Bensaid, 2003, p. 171) 
luego de la III Internacional, al amparo de una reflexión supuestamente "marxista". Indica también la sensibilidad de Marx por el problema campesino. Trae con mucha fuerza el famoso contenido de los borradores y de la carta a Vera Zasúlich, etc. Para Dussel, este "viraje"

(...) sólo se sitúa en el nivel histórico, concreto; nivel del desarrollo del discurso dialéctico. El nivel esencial, en donde se encuentra abstractamente el discurso de El Capital, no es cuestionado en absoluto. En realidad, no hay en él argumentos en pro o en contra de la cuestión de la "comuna rural rusa", porque ésta se sitúa en el nivel concreto o histórico de las condiciones de posibilidad de la aparición del capital. (Dussel, 1990)

Algo similar planteará E. P. Thompson, en sus estudios históricos y en lucha con interpretaciones mecanicistas, o estructuralistas del marxismo (particularmente la de Althusser). No necesariamente deben configurarse las clases en términos de la existencia de una clase (trabajadora en este caso) con una organización autónoma, una claridad en su proyecto político y la oposicion a una clase antagónica (capitalista), para poder interpretar ciertos períodos históricos (como el surgimiento y consolidación del capitalismo en el siglo XVIII inglés, pre-industrial), para poder hablar de lucha o de conflicto de clases, que es lo que intenta Thompson. De hecho el propio Marx lo hace de este modo en su conocido capítulo de el Libro I de El capital sobre la acumulación originaria. En este caso la clase funge más como categoría interpretativa que como una realidad efectiva, de una clase trabajadora organizada y con "moderna conciencia de clase". El otro plano de análisis sería en relación a un contenido histórico empíricamente observable. Sin embargo, como sostiene Badaró en relación a la obra de Thompson, en el caso del primer concepto ello afirma la "indisolubilidad de la relación entre clase y lucha de clases, sosteniendo inclusive la primacía del segundo término del par”. (Badaró, 2012: 88). En palabras del propio Thompson,

(...) la clase, en su sentido heurístico, es inseparable de la noción de "lucha de clases". A mi juicio, fue dada excesiva atención, frecuentemente de manera ahistórica, a la "clase", y muy poca, al contrario, a la "lucha de clases". En verdad, en la medida que es más universal, lucha de clases me parece ser el concepto prioritario. (Thompson, apud Badaró, 2012, p. 89)

En este sentido es posible afirmar que, en el período de formación del capitalismo y de la transición de la "subsunción formal" a la "subsunción real" del trabajo al capital (en los términos de Marx), que como sabemos tiene mucho que ver con la revolución industrial y con el paso de la manufactura a la gran industria, es posible hablar, según Thompson, de una "lucha de clases sin clases". Ello puede decirse en la medida que estas 
últimas no existían en su forma moderna en el siglo XVIII inglés, período analizado por Thompson, y que adquirirían dichas características de mayor organización y combatividad sobre los años 30 y 40 del siglo XIX, en los países centrales, y particularmente en Inglaterra. (cf. Badaró, 2012: 92). Pero sin embargo existían luchas de clase en el sentido moderno, en este caso en el siglo XVIII.

Algo similar podríamos decir en relación a las transformaciones del capitalismo tardío y fetiche de la actualidad, en la medida que parece desintegrarse la clase trabajadora en su fisonomía político organizativa e ideológica que se conformó durante el ciclo más propiamente industrial del capitalismo en la segunda mitad del siglo XX; sin embargo ni desaparecen las clases ni tampoco obviamente lo hace la lucha de clases en sí. $^{8}$

En todo caso lo que hoy parece estar sucediendo es lo que afirmaba el multimillonario Warren Buffet al New York Times en 2006: "Hay una guerra de clases, de acuerdo, pero es la mía, la de los ricos, la que está hacien do esa guerra, y vamos ganando".9

Incluso ciertos discursos con apariencias radicales desde posiciones de izquierda, dejan de tematizar la cuestión de las clases y sus luchas, confundiendo determinación económica con determinismo, y separando las críticas al capitalismo de cualquier protagonismo de los trabajadores en su superación, con efectos no menores sobre el pensamiento crítico. ${ }^{10}$

Hay algunas afinidades no menores entre el planteo de Thompson y la perspectiva

\footnotetext{
${ }^{8}$ En este sentido hay muchas similitudes entre Thompson y Bensaïd, a pesar de algunas críticas que éste último le dirige al primero: "Las clases no existen como realidades separables, sino sólo en la dialéctica de su lucha. No desaparecen cuando las formas más vivas o las más conscientes de la lucha se atenúan. Heterogénea y desigual, la conciencia es inherente al conflicto que comienza con la venta de la fuerza de trabajo y la resistencia a la explotación. Y que ya no cesa" (Bensaïd, 2003, p. 186).

9 http://www.elmundo.es/blogs/elmundo/billonarios/2014/06/10/palabra-de-warren-buffett.html, consultado el $14 / 5 / 18$.
}

\footnotetext{
${ }^{10} \mathrm{Al}$ respecto indica Aijaz Ahmad sobre este curioso procedimiento intelectual, no sin apelar a la ironía: "Lo que precisa ser justificado es otro tipo de ceguera, que rechaza ver que la mayoría de las cosas es una cuestión de clase. Ese rechazo en si mismo es, bien en el fondo, también una cuestión de clase. [...] Creo que la institucionalización de ciertos tipos de radicalismo han ido de manos atadas con una cierta asepsia de vocabulario, que en último análisis, es profundamente devastadora para el propio pensamiento. [...] la crítica al capitalismo es separada de cualquier validación directa de lo que podría substituirlo. Por ello, cuanto más antiburgués, mas anticolonialista, nos volvemos, menos hablamos sobre el socialismo como un horizonte viable. En el proceso, las críticas al capitalismo son separadas de cualquier necesidad de una política de la clase obrera" (Ahmad, 1999, p. 115, trad. propia).
} 
de Gramsci (incluso con el planteo de Bensaïd), innovando además con sus análisis históricos y en algunos campos relativamente poco explorados por el propio Marx y la tradición marxista anterior a Gramsci, en torno a la propia acción política, el Estado y la resignificación de la sociedad civil, la pedagogía, la cultura popular, la concepción de la revolución, el campo de los intelectuales, etc. Su comprensión del marxismo va en la línea de la "filosofía de la praxis". Nos interesa recuperar algunas de sus categorías, en particular las de hegemonía, de clases subalternas y de bloque histórico. Asumimos el desafío de no pensar dichos conceptos como construcciones ahistóricas, válidas para cualquier espacio/tiempo de la modernidad capitalista (lo que iría por otra parte contra el propio método gramsciano).

Para Gramsci la sociedad civil estará vinculada a la búsqueda de la hegemonía, entendida fundamentalmente como lucha ideológica, búsqueda de la dirección o "reforma intelectual y moral", en el marco de los llamados "aparatos privados de hegemonía". La misma se basa en la relativa autonomía que adquiere la sociedad civil en los tiempos de nuestro autor (escribiendo sus Cuadernos de la Cárcel en la transición de la década del 20 al 30 del siglo pasado), lo que implica su caracterización como una nueva esfera del ser social, estando asociada a los procesos de "socialización de la política".(Cf. Coutinho, 1999)

Por su parte el Estado en "sentido estricto", involucra los aparatos coercitivos, que remiten en lo fundamental a los aparatos de dominación, encarnados en grupos y estructuras burocrático-ejecutivos relacionados a las fuerzas armadas y policiales, y a la imposición y aplicación de las leyes. No existe una separación clara entre Estado en sentido estricto y sociedad civil (Estado "en sentido amplio" equivale a la suma de la sociedad política y la sociedad civil), aunque mantienen relativa autonomía. Distingue ademas entre las sociedades "orientales" y "occidentales"11:

En Oriente el Estado era todo, la sociedad civil era primitiva y gelatinosa; en Occidente, entre Estado y sociedad civil existía una justa relación y bajo el temblor del Estado se evidenciaba una robusta estructura de la sociedad civil. El Estado sólo era una trinchera avanzada, detrás de la cual existía una robusta cadena de fortalezas y casamatas. (Gramsci, 2003, p. 83)

Para Gramsci la hegemonía supone una "reforma intelectual y moral”, e incorpora la cuestión de una "voluntad colectiva nacional y popular". Pero ello no implica que la

${ }^{11}$ Dicho criterio no es en Gramsci estrictamente geográfico sino más bien analítico. 
hegemonía para Gramsci se desligue de la transformación de las relaciones de producción capitalistas, ni obviamente de las luchas de clase. Para Gramsci la esfera de la sociedad civil no es una esfera estrictamente autónoma, sino que está articulada de diferentes formas con el ámbito de la producción material y la reproducción de la vida social, y por lo tanto tampoco se desliga drásticamente de las luchas de las clases sociales fundamentales. Es más bien el escenario en que las luchas de clase toman forma políticoideológica.

Dicha hegemonía remite directamente al análisis de las "relaciones de fuerza" en una sociedad, desde una concepción procesual y conflictiva que desarrolla nuestro autor. Partiendo del primer nivel o momento, más propiamente estructural, es necesario abordar/analizar luego el plano más propiamente político, y fundamentalmente el plano de lo ético-político que supera el momento "económico-corporativo", siendo lo medular del plano de la hegemonía.

En síntesis, el concepto de hegemonía, sustentado en las transformaciones operadas en las formaciones capitalistas centrales y en las llamadas "sociedades occidentales", puede ser tanto referenciado para analizar las relaciones de hegemonía que establecen las clases y fracciones dominantes o hegemónicas, como para tematizar la búsqueda de una (contra) hegemonía, llevada adelante por las clases subalternas, los trabajadores o el pueblo trabajador. En dicho concepto incluye sin duda tanto a los trabajadores industriales del norte, como a los campesinos del sur de Italia, sectores de la pequeña burguesía, de los intelectuales, del clero, etc. Ello supone considerar (e impulsar) las luchas que se traban en el seno de los aparatos privados de hegemonía (que podríamos identificar como las que desarrollan diversos movimientos populares) junto con un conjunto de intelectuales orgánicos y grupos sociales aliados. Un componente central de la misma es la "reforma intelectual y moral", junto con la construcción de una "voluntad colectiva nacional-popular".

Sobre el concepto de bloque histórico, el mismo se vincula centralmente con su concepción de la hegemonía, tanto en el plano del análisis de la realidad social bajo una perspectiva de totalidad, como en sus implicancias políticas. Siguiendo a Coutinho, quien retoma el análisis de Hughes Portelli, el concepto tuvo en la obra de Gramsci dos acepciones fundamentales, aunque interconectadas. En primer lugar, se puede entender como la totalidad concreta formada por la articulación de la "infraestructura material" y 
las "superestructuras político-ideológicas", que reniega de las interpretaciones economicistas o reduccionistas presentes en diversas lecturas del "marxismo vulgar"12. En segundo lugar, como una alianza de clases bajo la hegemonía de una clase fundamental en el modo de producción, cuyo objetivo es conservar o revolucionar una formación económico-social existente. "La relación dialéctica se da en la medida en que la construcción de un "bloque histórico", en el segundo sentido, implica la creación de una nueva articulación entre economía y política, entre infraestructura y superestructura". (Coutinho, 1999: 153, trad. propia)

Por último, y a los efectos de nuestro análisis, resulta interesante en términos conceptuales aproximarse a la noción de sectores populares, y de sujetos colectivos populares, muy emparentado con el análisis de las clases subalternas en Gramsci, y con la articulación entre racionalidad, organización y cultura popular que plantea el pensador y militante italiano para el avance de un bloque histórico alternativo. El intelectual chileno Helio Gallardo, y en un nivel más político-concreto del análisis, nos advierte acerca de las relaciones entre pensamiento crítico, luchas sociales y sujetos colectivos populares. Para ello es importante partir previamente de su concepto de "lo popular":

Se considera social y objetivamente popular a los sectores sociales e individuos que son objeto de dominación estructural (y, bajo ciertas condiciones, también situacional). (...) Sectores sociales populares son, por lo tanto, objetivamente, todos los segmentos de la población, con sus diversidades internas, que soportan o sufren una dominación, o varias, dominación o dominaciones que ellos no pueden alterar liberadoramente sin organización, y movilización socio-política-cultural. (Gallardo, 2011, p. 78)

Para el autor, el "punto de partida" de cualquier actor popular o eventual sujeto colectivo popular,

(...) combina complejamente tanto factores de integración al sistema social (de dominación y de seguridad, básicamente), como dinámicas de desagregación y enfrentamiento (con sectores patronales, machistas, paternalistas, etc.) que constituyen el motor de una integración autónoma. La articulación positiva desde un punto de vista popular de estos factores desagregadores/integradores contiene como uno de sus factores un pensamiento crítico en situación. Sin embargo, para ser efectivamente crítico un pensamiento no puede encapsularse en el plano existencial,

\footnotetext{
${ }^{12} \mathrm{Si}$ bien Gramsci utiliza los conceptos (o las métáforas, según Thompson) de infraestructura y superestructura que plantea Marx en el Prólogo a la contribución a la crítica de la economía política, de 1859, el contenido que le da a los mismos se aleja de cualquier lectura mecanicista o economicista, y va en un sentido de totalidad y de mutua determinación y condicionamiento.
} 
sino que debe constituirse como un sentir-discernir o inteligir e imaginar sistémico. (Gallardo, 2011: 81)

Nos parece que esta conceptualización de los sujetos colectivos populares puede ser un punto de partida (aunque no el único obviamente) para superar algunas lecturas reduccionistas o eurocéntricas desde algunas perspectivas movimientos sociales, a la vez que para poder articular teóricamente las luchas de clases y las más vinculadas a lo sociopolítico. Al mismo tiempo permite conjugar dimensiones asociadas a lo racional, lo éticocultural, y el proyecto utópico de transformación social, y remite tambien a la historicidad de la conflictividad social y de las luchas sociales en Nuestra América.

\section{Clase, lucha de clases y luchas socio-políticas en el sistema-mundo capitalista y en Nuestra América}

Intentaremos ahora analizar someramente algunos trazos del desarrollo (y crisis) capitalista, con particular interés para el análisis de la formaciones sociales latinoamericanas y los sujetos colectivos populares en los procesos contemporáneos. Se trata de un nivel más concreto de análisis, en la medida que nos acercamos al análisis de procesos históricos concretos, lo que presupone una articulacion y entrelazamiento de elementos económicos, políticos, culturales, imaginarios, entre otros. En primer lugar se trata de superar algunos equívocos para poder intentar retomar mejor algunos aportes teóricos y políticos.

El sistema capitalista mundial, si tomamos por ejemplo los antecedentes de las teorías sobre el imperialismo de Lenin, Luxemburg, Hobson o Hilferding, o los aportes del "desarrollo desigual y combinado", al decir de Trotsky, planteará una tendencia al establecimiento de profundas jerarquías económicas y políticas entre regiones y países, en base a una particular división internacional del trabajo y el ascenso del mercado y el capitalismo global. La "dependencia"13 será posteriormente otra categoría central, tanto

\footnotetext{
${ }^{13}$ No debe entenderse la dependencia sólo como un problema de jerarquización entre regiones o entre diversas "composiciones orgánicas del capital", sino que la misma produce efectos igualmente jerarquizados y desintegradores al interior de los propios países dependientes, donde sectores hegemónicos alimentan dicha dependencia. Para ello la categoría de "colonialismo interno" de González Casanova (1970, entre otros) sigue siendo fundamental. A ello debe sumarse la creciente existencia de profundas desigualdades al interior de los países centrales producto de la modalidad especulativa y fetichista que adquiere en nuestro tiempo el capitalismo tardío. Pero ello no anula la importancia de las categorías de la dependencia. Por ejemplo en el caso de Ruy Mauro Marini (con sus categorías de superexplotación o subimperialismo), o en
} 
entendida como de unas regiones en relación a otras (incluyendo también la existencia de importantes diferencias al interior de las clases trabajadoras de los países dependientes, e inclusive de los capitales centrales y periféricos), como lo enfatizaron las teorías de la dependencia, entre otras interpretaciones (con diálogos con las perspectivas del desarrollo desigual, los análisis sobre el capitalismo monopolista, etc.). Otros aportes de autores como los S. Amin, I. Wallerstein o G. Arrighi irán en la línea del análisis de la acumulación capitalista en escala global, incorporando conceptos como los de semiperiferias, potencias hegemónicas, incorporando también algunos conceptos gramscianos para el análisis de las relaciones hegemónicas a nivel mundial. ${ }^{14}$

Esto lo podemos visualizar claramente al analizar el origen, desarrollo del capitalismo y la función que cumplió y cumple nuestro subcontinente a nivel de la acumulación originaria y su reproducción ampliada (o del moderno "sistema mundo" según Wallerstein). No parece necesario detenernos aquí en el carácter abiertamente "imperialista" o "colonialista" que adquirió la "conquista", y posterior incorporación a la dinámica del capitalismo y del capital, de Nuestra América. Sin embargo parece importante recordar que muchas de las modalidades de dominación y explotación implementadas, han tenido y tienen secuelas obvias en las trayectorias históricas y la realidad contemporánea de las propias configuraciones económicas, culturales y políticas en América Latina, así como de la propias "marcas" del capitalismo en su actual fase de "globalización". Vale citar al respecto el análisis de Aníbal Quijano:

La globalización en curso, es, en primer término, la culminación de un proceso que comenzó con la constitución de América y la del capitalismo colonial/moderno y eurocentrado como un nuevo patrón de poder mundial (...) América se constituyó como el primer espacio/tiempo de un nuevo patrón de poder de vocación mundial y, de ese modo y por eso,

el caso de nuevos desarrollos de la llamada "teoria marxista de la dependencia" como Marcelo Carcanholo o Jaime Osorio entre otros, que constituyen pistas fértiles de interpretación.

${ }^{14}$ En este sentido retomamos el análisis de David Harvey, en base a su postulación del desarrollo geográfico desigual del capitalismo y de su perspectiva desde un "materialismo histórico-geográfico": “(...) el capitalismo al mismo tiempo diferencia a los trabajadores unos de los otros, a veces alimentando antiguas distinciones culturales, relaciones de género, predilecciones étnicas y creencias religiosas. [...] El resultado de ello es la implantación en el paisaje geográfico del capitalismo de todo tipo de divisiones de clase, de género, y otras divisiones sociales. No es posible comprender divisiones como las que existen entre ciudades y periferias, o entre regiones como entre naciones, como residuos de algún orden antiguo. Y esas divisiones no desaparecen automáticamente, siendo activamente producidas por medio de los poderes diferenciadores de la acumulación del capital y de las estructuras de mercado. Proliferan lealtades territorialmente delimitadas que, en algunos aspectos, se fortalecen antes que desintegrarse, por medio de los mecanismos de la lucha de clases, así como a través de las propias actividades que desarrollan en favor de sí mismos tanto el capital como el trabajo" (Harvey, 2004b, p. 60-61: trad. propia). 
como primera id-entidad de la modernidad. [Para ello fueron necesarios dos procesos históricos convergentes:] la codificación de las diferencias entre conquistadores y conquistados en la idea de raza, es decir, una supuesta diferente estructura biológica que ubicaba los unos en situación natural de inferioridad respecto de los otros [y] la articulación de todas las formas históricas de control del trabajo, de sus recursos y sus productos, en torno del capital y del mercado mundial. (2002, p. 201-2)

En ese sentido, y siguiendo el análisis de Aníbal Quijano, "la humanidad actual en su conjunto constituye el primer sistema-mundo global históricamente conocido, no solamente un mundo como el que quizás fueron el chino, el hindú, el egipcio, el helénicorománico, el maya-azteca o el tawantinsuyano". Sin embargo el sistema actual, que comenzó a partir de la conquista, tiene en común tres elementos centrales que afectan la vida cotidiana de la totalidad de la población mundial: la colonialidad del poder, el capitalismo y el eurocentrismo (Quijano, 2002).

Ello implica que dicho desarrollo capitalista supone, al menos en las periferias del mismo, y en particular en Nuestra América, un desarrollo que está asociado inevitablemente a una matriz (neo) colonial del poder, al eurocentrismo (donde Europa quedará constituída progresivamente, - en buena medida por el aporte de mano de obra esclava e india y el flujo de excedentes hacia Europa que proporcionó América - como centro político, militar, económico e intelectual de dicho sistema). En dicho contexto la “clasificación racial” jugó y juega aún un papel central en dicha división internacional del trabajo y de las relaciones de poder a nivel mundial. ${ }^{15}$

Ahora bien, en este marco, ¿cómo pensar teóricamente la cuestión de las clases y las luchas de clase en relación a la cuestión de las experiencias de lucha y organización de los movimientos sociales, o más en particular desde las clases trabajadoras y subalternas en Nuestra América, desde una perspectiva de resistencia y construcción contrahegemónica?.

Como sabemos el propio concepto de movimientos sociales no ha estado exento de polémicas, ni de algunas presupuestos más bien eurocéntricas. Tanto en las perspectivas

\footnotetext{
${ }^{15}$ No podemos desarrollarlo aquí, pero cabe tomar ciertos recaudos frente a ciertas lecturas más contemporáneas, que retoman generalmente de forma parcializada diversos elementos del diagnóstico de Quijano, o que se inspiran en algunas perspectivas poscoloniales o de-coloniales, que abandonan su diálogo en el marco de una tradición crítica del materialismo histórico. Más bien se tiende a adherir a ciertas propuestas postestructuralistas, postmarxistas y/o de la "deconstrucción", las que, aún sostenidas por una necesaria crítica al eurocentrismo, pueden terminar alimentando propuestas relativistas o "culturalistas". Un ejemplo de ello puede verse en el colocar en un mismo lugar interpretativo y explicativo a las categorías de clase, nación, raza o género, por ejemplo, generalmente abandonando la categoría de lucha de clases.
} 
norteamericanas más clásicas que parten generalmente de una perspectiva de la "elección racional", como en lecturas europeas que priorizan las perspectivas de los "nuevos movimientos sociales", parece perderse dicha conexión con el plano conceptual de las luchas de clases. Tanto sea porque se entienda la acción colectiva desde una concepción más individual e instrumental (con énfasis en la dimensión institucional o de su influencia en el sistema político o de gobierno), o que se coloque el énfasis casi exclusivo en la cuestión de la novedad que implicarían los "nuevos" movimientos sociales (con reivindicaciones culturales, identitarias, entre otras), en relación a los "viejos" movimientos sociales pautados por la acción de los sindicatos modernos y los partidos de izquierda e incluso revolucionarios, dichas dimensiones son obscurecidas en el análisis. (cf. Gohn, 1997)

Otro tanto parece suceder con algunas perspectivas posestructuralistas y posmodernas, que pasan a tematizar la "deconstrucción", el poder a escala "microfísica" (olvidándose en ocasiones del poder estructural, y sobre todo del gran capital), exaltan la diferencia y el fragmento (más que la totalidad), estetizan las luchas, fragmentan y dispersan al sujeto, enaltecen el discurso y el lenguaje como mediación fundamental del ser social. (cf. crítica de Grünner, 2011). En no pocas ocasiones esto va en directa relación con un combate explícito o indirecto al marxismo, calificado como un "metarrelato", preso de la "metafísica" del progreso, incapaz de dar cuenta de la diversidad contemporánea, enterrado en el "baúl de la historia" luego de la caída de los "socialismos reales", etc. Uno de los abandonos más directos es sin duda la categoría de lucha de clases, lo que lleva a destacados intelectuales, inclusive del campo progresista y de izquierda, a postular las tesis del fin del trabajo, y por lo tanto también de las clases y de las propias luchas. Se continúa hablando de luchas, en ocasiones hasta el hartazgo, pero ello no se vincula con una crítica del orden social vigente desde una perspectiva de totalidad, y por lo tanto tampoco se plantean alternativas teóricas para pensar prácticas contrahegemónicas a dicho orden. Las luchas en todo caso aparecen más como parciales, desconectadas, como expresiones de una ampliación del campo de las libertades o de las “oportunidades", pero en no pocos casos aparecen como idénticas a sí mismas, como explicándose por sí mismas por su propia lógica inmanente, sin necesidad de vincularlas con otras luchas ni con las configuraciones del orden de dominación. Más bien lo que parece haber es una falta de tematización del propio capitalismo contemporáno, en la senda de la interpretación creativa de las categorías del propio Marx; y por lo tanto también existen serios déficits 
en la posibilidad de pensar y practicar alternativas y transformaciones substantivas de dicho orden. ${ }^{16}$

Sin embargo, también han podido observarse dificultades interpretativas en no pocos análisis que han pretendido inspirarse en la propia teoría social de Marx. Apelando a algunas lecturas economicistas o evolucionistas, sobre todo oriundas de las concepciones de la II o la III Internacional (ya estalinizada), el campo de las luchas sociales o de clases quedó reducido a un epifenómeno determinado por las contradicciones en el campo de la estructura económica. Se le quita por un lado significación a la propia lucha de clases, como configuradora de las posibilidades de constitución de las propias clases en pugna. Se prioriza el desarrollo de las fuerzas productivas, más que el análisis y el cambio en las propias relaciones sociales de producción. Se ha hablado mucho de condiciones objetivas y subjetivas para la revolución, o de "clase en sí" y "para sí”, y ello ha priorizado el desarrollo casi que evolutivo de las contradicciones en el campo económico como precondición para un "asalto" al poder político. En este sentido se ha descuidado la organización, la movilización, la formación, la lucha cultural, en espera de un momento cuasi milagroso donde emergería el potencial revolucionario de la clase obrera.

Otra distorsión ha sido el evolucionismo, muchas veces presente en las capas intelectuales y de izquierda de nuestras sociedades, de raíz eurocéntrica, que ha indicado que deben "quemarse" etapas para poder generar dichas condiciones objetivas y subjetivas. Ello supondría superar los resabios feudales de nuestras formaciones sociales (aún cuando nuestros países nunca hayan sido estrictamente sociedades feudales a la usanza europea), como precondición para la implantación de una revolución

\footnotetext{
${ }^{16} \mathrm{En}$ este sentido resulta sugerente la crítica que realiza Aijaz Ahmad a procesos que ocurren en algunas teorizaciones en el campo de lo "cultural" (entendida la cultura como no abstraída de sus relaciones con la "producción y reproducción material"), vinculadas a luchas que afirman la "separación de las identidades" al mismo tiempo que su "hibridez" y la "elección" de las mismas, asociada a algunos discursos sobre la "globalización", algunas luchas "identitarias" y a algunas perspectivas más recientes o despolitizadas de los "estudios culturales": "...quedamos impresionados con dos fenómenos paralelos que ocurren en la esfera ideológica de aquello que es llamado de estudios culturales. Existe la idea de la separación de identidades, cultural, étnica y nacional: una especie de diferencialismo implacable, de acuerdo con el cual no tengo permiso de alegar que puedo comprender su identidad, sino que debo simplemente respetar lo que sea que usted diga que son los requisitos de la misma. Según esa ideología, todos pueden elogiar las sólidas fronteras entre el self y el otro, denunciar lo que entienden como el "universalismo" del Iluminismo, racionalismo [...]. Al mismo tiempo y, de forma no poco frecuente, viniendo de las mismas personas, presenciamos también la idea de hibridez infinita, de las migraciones, de la elección de identidades alternativas o múltiples, como si nuevos selfs pudieran ser moldeados, instantáneamente a partir de cualquier arcilla en que podamos poner las manos, y como si culturas no poseyeran densidad e identidad históricas reales y pudiesen ser simplemente inventadas, sui generis, a partir de la circulación y maleabilidad globales de elementos recogidos en todo el mundo" (Ahmad, 1999, p. 113).
} 
"democrático-burguesa", previa a cualquier posibilidad de imaginar un tránsito al socialismo. El desarrollo de las revoluciones burguesas clásicas, como la inglesa, la francesa, o la norteamericana, ha sido tomado aquí como espejo. Pero ello supone en general un olvido sobre su carácter singular y no necesarsiamente reproducible en cuanto a dichas transiciones, sobre todo a la hora de pensar en otras modalidades más allá de las fronteras de dichos países o regiones

Otro problema ha sido desconocer la importancia de diversas reivindicaciones que parecen no ubicarse en el campo directo de la contradicción entre capital y trabajo. Reivindicaciones étnico-raciales, feministas y de género, de formas de producción más cooperativa o comunitaria (por ejemplo en el caso de los pueblos originarios), de tipo cultural, ecológicas, territoriales, incluso de luchas nacional-populares, entre otras, han sido vistas como secundarias, derivadas del conflicto central. En algunos casos han sido tachadas de resabios de modos de producción anteriores, de premodernas, o de reinvidicaciones pequeño burguesas o de las clases medias. Por lo tanto se han tendido a menospreciar, a postergar para luego de la toma del poder, en la medida que se entendía que ello implicaría perder el foco de las tareas urgentes, tanto desde el punto de vista táctico como más estratégico. La "dictadura del proletariado" (o el término que se prefiera) iría resolviendo estas cuestiones, y tampoco había que exagerar en cuanto a su importancia en relación a la lucha primordial, que seguía siendo entendida básicamente como la toma del poder político, y de forma generalmente explosiva, concentrado en el Estado burgués, para transformar la base material y la socialización de los medios de producción. ${ }^{17}$ Lo demás vendría por añadidura. En todo caso los estudiantes, mujeres, cooperativistas, grupos indígenas, campesinos y productores familiares, incluso comisiones vecinales o grupos culturales, podrían ser aliados (más o menos estables o coyunturales) de los trabajadores industriales (generalmente urbanos), pero nunca constituirían la vanguardia del proceso de cambios, el que por otra parte quedaba generalmente reducido al partido revolucionario (más bien de "pocos pero buenos", como fuera sostenido en algunas lecturas)

En este sentido otra cuestión no bien resuelta ha sido el cómo entender la

\footnotetext{
${ }^{17}$ Un excelente análisis de las distintas concepciones sobre el Estado y la revolución en Marx y Engels, así como en diversas tradiciones del campo marxista, pasando de teorias "restrictas" del Estado y concepciones "explosivas" de la revolución, hasta perspectivas, como la gramsciana o del último Poulantzas, que amplían la teoría del Estado y entienden el concepto de revolución como "proceso", puede leerse en Coutinho (1994).
} 
transición hacia una sociedad socialista/comunista o poscapitalista. Dichas concepciones han afectado también las posibilidades de construcción contrahegemónica de las clases populares. Muchas veces se ha contrapuesto de forma bastante maniquea la cuestión de la reforma a la de la revolución. Sin duda que Rosa Luxemburg en su clásico ensayo tenía razón en cuanto a cuestionar algunas lecturas reformistas de la transición, sobre todo de aquellas visiones evolucionistas etapistas que prácticamente colocaban el socialismo como una cuestión de reformismo gradual, con lo que el socialismo vendría casi que inexorablemente en la medida que el propio capitalismo agudizara sus contradicciones, y casi mágicamente se entraría en un proceso de transformación poscapitalista. En ese sentido se deja de lado cualquier posibilidad de ir construyendo o "prefigurando" ${ }^{18}$ el socialismo desde la práctica cotidiana de diversas luchas de clase y de los trabajadores. Con inspiración gramsciana Carlos Nelson Coutinho (1994), y también Lucien Goldmann, con inspiración austro-marxista (cit. en Bensaid, 2003, p. 287), hablan de la posibilidad de un "reformismo revolucionario", lo que obviamente complejiza esta cuestión. Enrique Dussel coloca una distinción entre reforma y transformación, más que propiamente entre reforma y revolución. ${ }^{19}$ Daniel Bensaïd, con aires benjaminianos y de un tiempo "mesiánico", entiende a la revolución como intempestiva, inactual, ajena a cualquier evolucionismo y a un tiempo lineal. ${ }^{20}$

Otro problema ha sido considerar, en algunas variantes de dicho marxismo vulgar,

\footnotetext{
${ }^{18}$ Según Ouviña, "la política prefigurativa desplegada por los movimientos populares latinoamericanos debe leerse en una clave bifacética, esto es, simultáneamente en términos de impugnación y autoafirmación propositiva. La creación de gérmenes o embriones de nuevas relaciones sociales, tiende a realizarse en el hoy, pasando de una inevitable lógica que Gramsci denominaba "luddista", (centrada en la impugnación de planes de ajuste estructural, o bien en la vocación "destituyente" predominante en no pocos movimientos populares durante los años noventa), a una que cede paso a la edificación prefigurativa, sin esperar para ello la "conquista del poder" como algo inmediato (2013, p. 91)

${ }^{19}$ Para Dussel la oposición central es entre reforma y transformación, donde la revolución supone una modalidad posible, la de la transformación radical, pero donde también se reconoce la importancia de las transformaciones parciales. Lo importante aqui parece ser nuevamente la perspectiva estratégica de transformación social en la que se inscriben las transformaciones parciales. (2006)

20“Plena de recién llegados y viejos espectros, la revolución es, por el contrario, el acontecimiento por excelencia. Inactual, intempestiva, ella en suma "nunca tiene lugar en el presente". Siempre demasiado temprano $y$ demasiado tarde. La revolución merodea en los márgenes de lo político. Más allá se extienden tierras inciertas, regiones sin nombre, que exceden los límites de lo pensable. [...] Como derrocamiento, la revolución es un acto político. Como disolución, es un proceso social. Es simultáneamente una revolución del tiempo largo, intelectual y moral, que mina lentamente el fundamento de los imperios, y una revolución insurrecta en el asombro de su propia irrupción. Unidad de rupturas y continuidades, de tradición y de auténtica novedad, combinación de tiempos mezclados, tra el límite donde lo pensable se muere en la incertidumbre de la elección. [...] Es mundo es el de "las explosiones, los cataclismos y las crisis". Donde las contradicciones se resuelven en la violencia de la decisión" (Bensaïd, 2003, p. 149-150).
} 
de forma estrecha a la propia clase trabajadora en nuestros países. Se ha tendido a asimilar a las clases trabajadoras con la clase obrera ${ }^{21}$, en su versión de la gran industria. Y como sabemos, el proletariado industrial, si nos remitimos a los países del llamado Tercer Mundo, o de Nuestra América en particular, prácticamente siempre ha sido históricamente minoritario en relación al conjunto de las clases trabajadoras, populares o subalternas (en términos de Gramsci).

Esto incluso puede decirse de los países que han tenido un desarrollo industrial más fuerte, durante el período más propiamente desarrollista del capitalismo dependiente, como Argentina, Brasil y México. ${ }^{22}$ Por su parte los campesinos y las comunidades indígenas ha tendido a verse más bien como un grupo conservador, hasta reaccionario. Lo mismo podría decirse de los levantamientos de los esclavos, de las rebeliones indígenas, de las montoneras en los países del sur, etc., en la medida que no se habrían articulado con la resistencia a la conformación moderna del capitalismo.

Por su parte los sectores populares que han engrosado las periferias de las grandes ciudades, en busca de nuevas oportunidades de vida, y cuyas condiciones materiales de vida los ubican claramente en el campo de las clases subalternas, también han sido considerados, generalmente de forma despectiva, como "lúmpen proletariado", como una variante de las llamadas "under class", o incluso como presas o "carne de cañón" del "caudillismo" o del clientelismo de los partidos más conservadores o del poder religioso. Y con ello también se han desconsiderado otras dimensiones que atraviesan a las clases populares en América Latina, compuestas mayoritariamente por mujeres, jóvenes, población indígena, mestiza, negra, mulata ${ }^{23}$; también se ha despreciado la religiosidad y la cultura popular, sus lenguas, sus tradiciones de organización productiva y social, etc.

\footnotetext{
${ }^{21}$ Pero incluso, según la lectura de Marcelo Badaró y Daniel Bensaïd que resulta muy convincente, existe una confusión en las lenguas neolatinas, que lleva a asimilar la clase trabajadora a la clase obrera. Sin embargo "classe ouvriere" no es la traducción automática de "working class". En este sentido, argumentan los autores, el sentido que Marx le atribuye al concepto es mucho más el de clase trabajadora que el de clase obrera, o industrial. Marx habla fundamentalmente del proletariado o proletarios, lo que según Bensaïd resulta incluso más riguroso y abarcativo que el de clase obrera. (Badaró, 2012, p.66)

${ }^{22}$ En estos últimos años el proletariado industrial ha crecido sin dudas en la región centroamericana y en México, al amparo de la producción de las "maquilas" para la economía norteamericana y de los tratados de libre comercio con América del Norte. Pero se trata sin duda un proletariado altamente fragmentado, reprimido y precarizado.

${ }^{23}$ Ello obviamente ha variado según el país y la región. Tampoco debe olvidarse que la inmigración europea que recibieron nuestros países también tenemos una fuerte presencia de sectores populares, artesanos y trabajadores de la ciudad y el campo, con ideologías anarquistas, socialistas y mutualistas, y en no pocas ocasiones se vinculaba a sectores perseguidos política o religiosamente, etc.
} 
Varias categorías se han ensayado o aplicado para intentar caracterizar sus condiciones materiales y de reproducción: desde grupos marginados, informales, ejército industrial de reserva, excluídos, precariado, subproletariado, pobretariado, sobre-explotados, etc ${ }^{24}$. En la medida que tendía a desconsiderarse su inclusión en el campo de las clases populares, también tendieron a ser invisivilizados como agentes potencialmente revolucionarios; en todo caso podían asumir alguna función yendo como "furgón de cola" de la clase obrera industrial y de los partidos de vanguardia.

Sobre finales del siglo XX, muchas de estas expresiones populares, articuladas bajo diversas resistencias, rebeliones, movimientos sociopolíticos o potencia "plebeya", contribuirán sin duda a cuestionar los efectos de las políticas neoliberales, y sumarán al giro a la izquierda que se produce en la región, vinculándose con la emergencia de los gobiernos progresistas, nacional-populares o del llamado socialismo del siglo XXI. Esto también ha tenido consecuencias para muchos de dichos sectores y movimientos populares, que parecen ir en la dirección de un agotamiento o neutralización de muchas de dichas luchas (cf. Falero, 2014). Sabemos que este ciclo hoy se encuentra en un impasse, y que las fuerzas de la derecha han avanzado significativamente en la región, muchas veces al amparo de los recientes golpes institucionales como el de Honduras, Paraguay o Brasil, o por victorias electorales como las de Macri en Argentina, o del regreso de Piñera en Chile. Han incluso avanzado en relación a una disputa hegemónica travada en el seno de la sociedad civil (en el sentido gramsciano), tanto en sus dimensiones ético culturales (por ejemplo en la lucha contra el aborto o en la disputa contra la mal llamada "ideología de género", y avanzando claramente bajo formatos neofascistas y conservadores), como socio-políticas, por ejemplo en las movilizaciones frente al gobierno de Chávez o de Maduro en Venezuela, en las de sectores medios altos y empresariales luego de los episodios de 2013 en el Brasil y el proceso de golpe parlamentario contra el gobierno de Dilma Rousseff en 2016, replanteando una agenda claramente neoliberal para nuestros países, etc. ${ }^{25}$

Luego de este rápido repaso, nos interesa enfocar algunas primeras conclusiones

\footnotetext{
${ }^{24}$ Escapa naturalmente a los objetivos de este trabajo poder realizar un análisis y balance de estas distintas categorizaciones sobre los sectores populares en América Latina.

${ }^{25}$ No es el objeto de este artículo analizar este relacionamiento y vinculación entre movimientos populares y gobiernos progresistas, y tampoco la discusión abierta de si estamos frente a un agotamiento, crisis terminal o redefinición de este ciclo progresista en América Latina. Desarrollamos algunos argumentos en relación a estas temáticas en Casas, 2017, mímeo.
} 
de lo planteado, así sean provisorias. Una primera lectura indica sin duda una diversidad de actores, de luchas, de contradicciones y de conflictos sociales que expresan las particularidades de nuestras formaciones sociales, y de las formas de protesta, organizativas y de las demandas colocadas por muchos de estos movimientos. Como indicábamos anteriormente, dicha diversidad de expresiones ha llevado a algunas interpretaciones desde las ciencias sociales a colocarlas por fuera de las más clásicas configuraciones de las clases modernas y de sus luchas en las sociedades capitalistas. ${ }^{26}$

No es nuestra intención aquí hacer un balance de otras contribuciones en el plano del pensamiento y la teoría social en América Latina en relación a estas cuestiones. Solamente queremos decir que se fueron elaborando otras interpretaciones críticas en relación a aquellas dominantes. Por ejemplo desde las contribuciones originales del estructuralismo cepalino se pondrán algunos nuevos parámetros para pensar la cuestión de la explicación del subdesarrollo, y sobre todo lo harán las teorías de la dependencia ${ }^{27}$ al mostrar el carácter plenamente capitalista de nuestras formaciones sociales y la necesidad de avanzar en una estrategia antiimperialista y de carácter socialista en nuestros países. Ya en los años 20 en el Perú Mariátegui había aportado sus contribuciones originales desde un marxismo crítico en América Latina, que incorporaba claramente la cuestión de la participación indígena en los procesos revolucionarios en nuestros países. Otras contribuciones desde tradiciones vinculadas al marxismo como los propios desarrollos de las teorías sobre el imperialismo o el capitalismo monopolista, desde la historiografía o la sociología crítica (en Brasil son destacables por ej. los aportes de Caio Prado Junior y Florestan Fernandes), o incluso desde la teología y la filosofía de la liberación, harán lo propio para desmontar aquellas lecturas modernizadoras, funcionalistas o etapistas. Naturalmente los propios procesos revolucionarios vividos en América Latina, particularmente la revolución mexicana y la boliviana, pero sobre todo la revolución cubana al triunfar en 1959, tendrán efectos nada menores en algunas de dichas

\footnotetext{
${ }^{26}$ Un ejemplo de esto fueron algunas interpretaciones de la sociología de Alain Touraine sobre América Latina que, al hablar de los nuevos movimientos sociales emergentes en América Latina sobre fines del siglo $\mathrm{XX}$, ubicaron dicho campo de conflictos por fuera del tradicional campo de la lucha moderna de clases. Desde esa lectura no habría propiamente clases sociales como en el capitalismo central, y por lo tanto las dinámicas del conflicto social estarían atravesadas por otro tipo de demandas más volcadas hacia la identidad, lo cultural, etc., y ya no se explicarían en función de las contradicciones clasistas del capitalismo (cf. Bensaïd, 2003, p. 267).

${ }^{27}$ Decimos en plural porque dichas perspectivas se desarrollaron en distintas direcciones teóricas, desde lecturas más neoweberianas como las de Cardoso y Faletto, hasta versiones más cercanas al marxismo como las de Theotonio Dos Santos, Vania Bambirra y sobre todo Ruy Mauro Marini. Ver al respecto Martins, 2006.
} 
nuevas lecturas teóricas y políticas. Otras influencias vendrán también del proceso de la Unidad Popular en Chile, así como de la revolución nicaraguense. Dicho proceso de renovación teórica desde una teoría crítica en América Latina, influenciada por una relectura desde un marxismo crítico y por las luchas de los movimientos populares y los procesos revolucionarios, será atacado en buena medida por los procesos dictatoriales y las doctrinas de "seguridad nacional". El mismoreemergerá sólo más recientemente, incluyendo nuevos aportes, a comienzos del siglo XXI en nuestra región, vinculado también a un ciclo ascendente de resistencias y movilizaciones del campo popular, en particular frente a las políticas de ajuste estructural del capitalismo de fin de siglo. ${ }^{28}$

Tomando en cuenta algunos de estos aportes en la tradición de un marxismo crítico renovado, es que podemos efectuar un ejercicio de crítica en relación a algunas de las lecturas anteriormente indicadas sobrelos procesos socio/históricos reseñados en el apartado anterior. Como decíamos, aparece una diversidad de configuraciones más estructurales de las clases trabajadoras en América Latina, que, desde un punto de vista más "esencial", se sitúan en un campo de relaciones de subordinación, resistencias y tentativas de construcción de alternativas sociales que no pueden desligarse del plano del control y de la dominación sociometabólica del capital, al decir de Mészáros (2002). Frente a sociedades que han sido estructuralmente moldeadas por la lógica del capital y del capitalismo, que en nuestros países ha tomado una forma básicamente colonial primero y luego dependiente, no es posible abstraernos de estas determinaciones a la hora de analizar a los movimientos sociales y populares en nuestra región. Esto incluso lo podemos ver no sólo en cuanto a la permanencia (aunque relativamente debilitada) de formas de organización de trabajadores generalmente más estables y/o con mejores condiciones de trabajo (tanto a nivel público como privado) en sindicatos y centrales de trabajadores (lo que varía mucho según el contexto histórico y los países analizados), sino también en relación a la autodenominación de muchos de estos movimientos populares, al identificarse como "trabajadores sin" tierra/techo/trabajo o desocupados, etc. $\mathrm{Al}$ mismo tiempo otros movimientos tienen una fuerte presencia vinculada al mundo del trabajo, por ejemplo en relación a la economía social, la economía solidaria, el movimiento de empresas recuperadas y autogestionadas por sus trabajadores, etc. El caso del

\footnotetext{
${ }^{28}$ Desarrollamos muchas de estas contribuciones para un marxismo crítico en América Latina en nuestra Tesis de Doctorado (UFRJ, Brasil) titulada Marxismo y pensamiento crítico en América Latina: dependencia, integración y crítica de las utopías. Mímeo, 2007.
} 
movimiento cooperativo de vivienda en Uruguay, si bien ha colocado el foco en la vivienda popular en una modalidad colectiva de construcción, propiedad y uso, también se ha nutrido fuertemente de trabajadores vinculados a diversas experiencias gremiales y de organización sindical. ${ }^{29}$

\section{Consideraciones finales}

Tomando en cuenta estos elementos, es que podemos pensar en la constitución (al menos desde el punto de vista teórico-político) de "bloques históricos" (en sentido gramsciano) desde el campo popular, o de sujetos colectivos populares que pretenden luchar de forma articulada contra distintas formas de dominación y explotación. Si prevalecen las demandas aisladas, o incluso intereses y demandas "hegemonistas" de algunos grupos (que no deben confundirse con la búsqueda de hegemonía), obviamente tendremos un campo de mucha mayor fragmentación, lo que termina obviamente por reproducir y perpetuar el orden de dominación ${ }^{30}$; si las articulaciones se reducen a una

\footnotetext{
${ }^{29}$ Pero incluso si pensamos en actores que en una visión más superficial podríamos ubicar como teniendo un peso relativo en relación al mundo del trabajo y las luchas más tradicionales de clase, ello no se condice con la importancia socio/política que sí han tenido en los procesos sociales y revolucionarios en América Latina. Por ejemplo podemos citar el caso del movimiento campesino indígena que ha tenido una partipación más que destacada en amplios procesos de movilización y de cambio en la América Latina reciente, en particular desde la emergencia zapatista en 1994 (aunque sin el objetivo explícito de apuntar a la toma del poder del Estado), con el caso ecuatoriano en los orígenes del proceso de la revolución ciudadana y en el proceso boliviano con la creación del MAS y la Asamblea Constituyente, aunque también tuvo un protagonismo importante en la alianza que lleva a Lugo a la presidencia del Paraguay; las movilizaciones contra el ALCA que es abandonado en el 2005 en Mar del Plata; puede decirse algo similar de la importancia de los movimientos estudiantiles en rechazo a las privatizaciones oriundas de la dictadura neoliberal en el Chile reciente, que han logrado niveles muy significativos de movilización y protesta, así como de conquistas parciales de sus objetivos; en los movimientos de resistencia a las privatizaciones en el Uruguay de los 90 a partir de diversas coaliciones socio-políticas; en los movimientos vinculados a la demarcación de tierras quilombolas en Brasil, o en cuanto al sistema de cuotas en las universidades, o en los movimientos de la población afrodescendiente en Colombia, por ej.; en los movimientos de derechos humanos en Argentina pero tambien parcialmente en Uruguay, que han logrado colocar la cuestión de la lucha contra la impunidad en la agenda de los gobiernos de la región; en diversos movimientos ecologistas que han resistido a la expansión de la frontera minera, del frakking, del agronegocio, etc, incluso frente a capitales de origen indio, chino o canadiense; en las amplias movilizaciones de grupos feministas y de mujeres como en el caso del "Ni una menos" o en las luchas por la despenalización del aborto; otros casos se vinculan a movimientos de neto perfil popular, que pueden tener expresiones orgánicas o no, o que se manifestan esporádicamente en cuanto a su incidencia política, por ejemplo en el respaldo a distintos gobiernos progresistas de la región, fue claro el apoyo de las barriadas venezolanas de Caracas para frenar el golpe de Estado contra Chavez en el 2002, han sido también dichas bases sociales que han respaldado a los gobiernos del PT en Brasil, etc.

${ }^{30}$ Como indica Hernán Ouviña: "Más allá de pensar desde y a partir de lo plural, para muchas organizaciones populares la cuestión gira alrededor de un dilema pendiente aún de resolver: cómo dar el paso de la multiplicidad de sujetos en lucha, hacia la proyección de un sujeto múltiple que, no obstante, continúe estando habitado por la diversidad, esto es, sin que se imponga la homogeneización como parámetro de
} 
especie de diálogo "multisectorial" o en acuerdos puntuales, pueden haber ciertos avances pero no lograr constituir verdaderos sujetos colectivos que apuntalen procesos de resistencia, organización y lucha antisistémica; si se avanza en términos de la constitución de sujetos colectivos populares que se ubiquen en función de diálogos genuinos, solidaridades y acciones compartidas (incluyendo obviamente el campo de la producción, distribución y consumo), con análisis y ejes programáticos conjuntos de actuación (lo que obviamente no quiere decir unanimidades ni la no expresión del disenso), incluyendo mediaciones organizativas y partidarias de nuevo tipo que no subordinen la acción político institucional a la organización, movilización y autonomía popular, parecería que podriamos transitar hacia la constitución de bloques contrahegemónicos capaces de disputar hegemonía y construcción de nuevas relaciones de poder y saber.

Al decir de Ouviña se trata de

(...) ejercitar una dinámica pendular y complementaria que, por un lado, apueste al fortalecimiento interno de los espacios de construcción prefigurativos (a través de la creación y consolidación de instancias de autogobierno conectados orgánicamente a la vida cotidiana, como "modus vivendi") y, por el otro, intente generar de manera constante ámbitos de coordinación, irradiación y confluencia, en niveles que simultáneamente excedan y contengan la dimensión local y regional de las luchas. La cuestión, al parecer, estriba en cómo resignificar esta certera dinámica bifronte, creando alianzas y redes pluri-identitarias y cambiantes, en donde ninguna de las fuerzas socio-políticas que las conforme tenga más derecho a hegemonizar, que el que le da su comportamiento concreto en cada coyuntura histórica". (Ouviña, 2013: 93-94)

Las clases y las luchas de clases no han desaparecido, en la medida que tampoco lo ha hecho el capitalismo como sistema cada vez más global pautado por el orden sociometabólico del capital, con características que apuntan en el sentido de un empeoramiento de las condiciones de vida de amplias mayorías a nivel mundial, junto a procesos que siguen alentando la concentración y centralización del capital, donde cada vez un menor grupo de bancos y grandes corporaciones trasnacionales controlan la producción, circulación y distribución de las mercancías a nivel global, así como tienden a capturar las lógicas de los organismos internacionales y de los Estados nacionales, impactando fuertemente en la calidad de nuestras democracias. Esto además ha traído aparejado innumerables y devastadores efectos ambientales, a la par que ha tenido un

coordinación y confluencia de experiencias disímiles y ricas en sí mismas" (2013, p. 94). 
impacto directo en las condiciones de producción y reproducción de las clases trabajadoras a nivel global. Ello lleva a un horizonte de barbarización capitalista cada vez más exacerbado, llevando a una "crisis estructural del sistema de capital" (Mészáros, 2002), a una lógica de "acumulación por desposesión" (Harvey, 2004), entre otros diagnósticos de la situación actual. Ello implica que también tengamos el desafío de dar cuenta de las transformaciones operadas por estos procesos en las diversas clases sociales, en las manifestaciones de las luchas de clases y de las luchas socio-políticas y éticoculturales en Nuestra América. Y un desafío teórico de la mayor importancia reside tambien en articular el plano más teórico-esencial, con el imprescindible análisis histórico-político, contribuyendo a análisis concretos y coyunturales que permitan hacer avanzar el atender a los desafíos del presente.

Todo ello obviamente no supone que las alternativas poscapitalistas estén a la orden del día. Flaco favor le haríamos al sistema de dominación si dejáramos de dejar de analizarlo tanto en su globalidad como en sus impactos y manifestaciones a nivel regional y local, junto con sus impactos en la vida cotidiana, desde una perspectiva de totalidad. Para ello el marco categorial del marxismo original sigue siendo fundamental, aunque seguramente no suficiente, para dar cuenta de las transformaciones en curso e iluminar posibles realidades alternativas. Pero tampoco será posible construir dichas alternativas sin el protagonismo amplio y articulado de diversos sujetos colectivos populares, como expresiones más directas de dichas luchas de clases y conflictos socio-políticos que instaura el orden del capital, las que incluyen un conjunto muy diverso de experiencias, manifestaciones, resistencias y autonomías, en escalas tanto locales como regionales y globales. $^{31}$

Pretender que la lucha de clases, tanto en el plano de la interpretación de los

\footnotetext{
${ }^{31}$ En este sentido es sugerente lo afirmado por Harvey en relación a la vigencia del marxismo y la necesidad de continuar trabajando en la permanente síntesis de múltiples luchas, apelando a la defensa y vinculación entre intereses de luchas "particulares", "adecuadamente unificados" y el "interés general": "Una de las fuerzas históricas del marxismo ha sido su compromiso con la síntesis de diversas luchas cuyas metas son múltiples y divergentes en un movimiento anticapitalista más universal. La tradición marxista tiene aquí una inmensa contribución que dar, pues es pionera en lo tocante a los instrumentos por medio de los cuales identificar puntos comunes en medio a multiplicidades y diferencias (a pesar que, en algunas ocasiones, haya absorvido con demasiada rapidez estas últimas en aquellas). El trabajo de síntesis de las múltiples luchas hoy existentes ha de ser permanente, pues los campos y terrenos en que dichas luchas se traban, y las cuestiones a las que responden, están en perpetuo cambio, acompañando las alteraciones de la dinámica capitalista y de las condiciones globales asociadas. La frase de Raymond Williams acerca de la "defensa de ciertos intereses particulares, adecuadamente unificados" como manera de brindar bases al "interés general"es por lo tanto la tarea esencial a ser empreendida" (Harvey, 2004b: 102-3).
} 
procesos sociales como en cuanto a la construcción de alternativas poscapitalistas, no tenga expresiones vinculadas a lo étnico-racial, lo nacional-popular, el género, la lucha anticolonial o el campo de la cultura, sería un error teórico y político tan importante, como pensar que dichas dimensiones del conflicto social no están articuladas, por diversas mediaciones, con las luchas de clases y las clases tal cual se van configurando, y también transformando, en el sistema capitalista. Pensar que privilegiando alguna de estas dimensiones por sobre otras como elementos explicativos del conflicto social, amparadas en interpretaciones fragmentadas de la realidad social, o desatar una lucha por dichos conflictos en términos identitarios, es abonar la fragmentación y la reproducción barbarizante del orden de dominación. Tampoco se trata de pensar en luchas fundamentales y en luchas secundarias, pero tampoco de entender que todas tienen el mismo poder explicativo y transformador, colocándolas en un lugar de relativa igualdad o indeterminación en relación a las demás. Muchas luchas o resistencias pueden ser absorvidas por el sistema, y de hecho muchas lo han sido a lo largo del desarrollo capitalista, aún sin alterar los fundamentos de su reproducción sociometabólica. En este sentido abandonar la lucha de clases, tanto teórica como políticamente, significa también abrir el paso a la imposibilidad de crítica y de transformación hacia una sociedad poscapitalista.

\section{Referencias bibliográficas}

AHMAD, A. (1999): Pós-modernismo y movimientos populares. Problemas de clase y cultura, en WOOD, E.M. y BELLAMY FOSTER, J.: Em defesa da história. Marxismo e pósmodernismo. Rio de Janeiro: Zahar Ed., pp. 107-122.

BADARÓ MATTOS, M. (2012): E.P. Thompson e a tradicao de crítica ativa do materialismo histórico. Rio de Janeiro: Ed. UFRJ.

BENSAÏD, D. (2003): Marx intempestivo. Grandezas y miserias de una aventura crítica. Buenos Aires: Herramienta.

CASAS, A. (2017) Hegemonía, revolución pasiva y bloques históricos en el Uruguay contemporáneo: una primera aproximación, ponencia presentada en el marco del XII Congreso Nacional de Trabajo Social: “Transformaciones sociales, protección social y Trabajo Social”, mímeo. Montevideo el 6-7 julio. 
contemporáneo y en la actual coyuntura en AAVV: Sujetos colectivos populares, disputas hegemónicas y Trabajo Social. Montevideo: UR. Cuaderno de investigaciones n 1.(en prensa)

(2007): Marxismo y pensamiento crítico en América Latina: dependencia, emancipación y crítica de las utopías. Rio de Janeiro: UFRJ. Tesis de Doctorado en Servico Social. Mímeo.

COUTINHO, C. N. (1999): Gramsci: Um estudo sobre seu pensamento político. Rio de Janeiro, Civilizacao Brasileira. Nova edicao revista e ampliada.

(2006): Intervencoes. O marxismo na batalha das ideias. Sao Paulo: Cortez. (1994) Marxismo e política. A dualidade de poderes e outros ensaios. Sao Paulo:

Cortez.

DUSSEL, E. (1990): El último Marx (1863-1882) y la liberación latinoamericana. Un comentario a la tercera y a la cuarta redacción de "El Capital". México: Siglo XXI Iztapalapa.

(2006): 20 Tesis de Política. Siglo XXI, Centro de Cooperación Regional para la Educación de adultos en América Latina y el Caribe.

Siglo XXI.

(1985): La producción teórica de Marx. Un comentario a los Grundrisse. México:

FALERO, A. (2014) "Del cambio a la contención del cambio: período bisagra en América Latina? En ACOSTA, Y.; CASAS, A.; MAÑAN, O.; RODRÍGUEZ, A.; ROSSI, V.: Sujetos colectivos, Estado y capitalismo en América Latina. Perspectivas críticas. Montevideo: Espacio Interdisciplinario, UR. pp. 19-32

GALLARDO, H. (2011): "Pensamiento crítico y sujetos colectivos en América Latina" en ACOSTA, Y.; FALERO, A.; RODRÍGUEZ, A.; SANS, I.; SARACHU, G. (coords.) (2011):

Pensamiento crítico y sujetos colectivos en América Latina. Perspectivas interdisciplinarias. Montevideo:Trilce-Espacio Interdisciplinario UR, pp. 77-95.

GOHN, M.G. (1997) Teorias dos movimentos sociais: paradigmas clássicos e contemporâneos. São Paulo: Loyola.

GONZÁLEZ CASANOVA, P. (1970): Sociología de la explotación. México: Siglo XXI, 2ª ed.

GRAMSCI, A. (1998): Cartas desde la cárcel. Buenos Aires: Nueva Visión.

(2003): Notas sobre Maquiavelo, sobre la política y sobre el Estado moderno.

Cuadernos de la Cárcel. Buenos Aires: Nueva Visión.

GRÜNNER, E. (2011): Los avatares del pensamiento crítico, hoy por hoy. En GRUNNER, E. (coord.) Nuestra América y el pensar crítico. Fragmentos de Pensamiento crítico de Latinoamerica y el Caribe. Buenos Aires: Clacso.

HARVEY, D. (2004): El “nuevo” imperialismo. Sobre reajustes espacio-temporales y 
acumulación mediante desposesión. Parte I. Buenos Aires: Revista Herramienta N² 27, octubre.

(2004b): Espacos de esperanca. Sao Paulo, 2004: Loyola, 4ta ed..

MARTINS, C. E. (2006): O pensamento latino-americano e o sistema mundial en AAVV: Crítica y teoría en el pensamiento social latinoamericano. Buenos Aires, CLACSO, Colección Becas de investigación, pp. 153- 216.

MARX, K. Y ENGELS, F. (1969): Manifiesto del Partido Comunista. En Marx y Engels: Obras Escogidas. Moscú: Ed. Progreso.

MARX, K. (1984): Elementos fundamentales para la crítica de la Economía Política (Grundrisse). 1857-58. Volumen I. México, Siglo XXI ed., 13era ed.

MÉSZÁROS, I. (2002): Para além do Capital. Rumo a uma teoria da transição. São Paulo: Boitempo-Unicamp.

OUVIÑA, H. (2013) La política prefigurativa de los movimientos populares. México: Acta Sociológica 62. pp. 77-104.

QUIJANO, A. (2002): Colonialidad del poder, eurocentrismo y América Latina. En LANDER, E. (comp.) La colonialidad Del saber: eurocentrismo y ciencias sociales. Perspectivas latinoamericanas. Buenos Aires, CLACSO. pp. 201-243.

ROSDOLSKY, R., (1978): Génesis y Estructura de El Capital de Marx (estudios sobre los Grundrisse). México: Siglo XXI.

THWAITES REY, M. (2004): La autonomía como búsqueda. El Estado como contradicción. Buenos Aires: Prometeo.

ZIZEK, S. (2005): Multiculturalismo, o la lógica cultural del capitalismo multinacional. pp. 137-188, en JAMESON, F., ZIZEK, S.: Estudios culturales: reflexiones sobre el multiculturalismo. Buenos Aires: Paidós, 3era reimp. Con estudio introductorio de E. GRUNER. 